
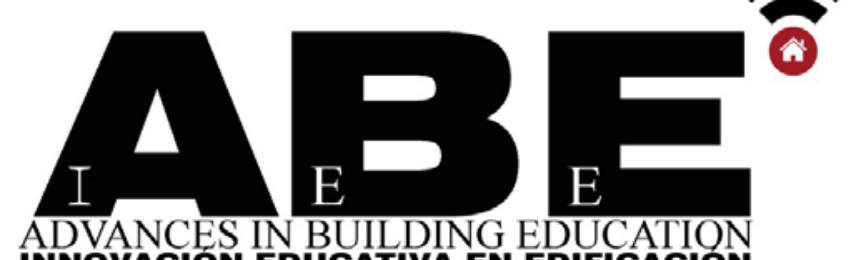

\title{
Enseñanza de la filosofía de Lean Construction en la formación de Ingenieros Civiles: Una actualización del diseño del curso
}

Teaching Lean Construction for undergraduate Civil Engineering students: An update on course design

Sofia Villagarcia Zegarra ${ }^{1 *}$, Xavier Brioso ${ }^{2}$,

1 Profesora Auxiliar, Grupo de Investigación GETEC, Pontificia Universidad Católica del Perú, svillagarcia@pucp.pe ${ }^{2}$ Profesor Principal, Grupo de Investigación GETEC, Pontificia Universidad Católica del Perú, xbrioso@pucp.edu.pe

\section{TITULARES}

- Enseñanza de Lean Construction.

- Actualización del diseño del curso.

- Utilización de herramientas en la industria de la Arquitectura, Ingeniería y Construcción

\section{HIGHLIGHTS}

- Teaching Lean Construction

- Update on course design

- Using tools in the Architecture, Engineering and Construction industry 


\section{RESUMEN}

Este artículo tiene como objetivo resaltar la importancia de la enseñanza de la filosofía Lean Construction para complementar la formación de alumnos de pregrado de la carrera de ingeniería Civil y dar pautas para la actualización del diseño del curso sabiendo que para que sea efectivo el aprendizaje este debe estar relacionado a la solución de problemas que suceden en el día a día en los proyectos de construcción y teniendo en cuenta que los estudiantes aún no cuentan o tienen poca experiencia profesional. Por ello, el uso de herramientas donde el alumno pueda entender la problemática y poder desarrollar el pensamiento analítico y critico es indispensable. En ese sentido, una herramienta didáctica muy útil son los estudios de caso y ejemplos reales de problemas que suceden en las obras ya que lleva al alumno a entender a través de casos los problemas que suceden durante el desarrollo y ejecución de proyectos y como se pueden plantear soluciones a través de la aplicación de conceptos Lean. Es importante mencionar que la aplicación de la filosofía Lean tanto en el Perú como en el mundo está ganando terreno rápidamente por ello el introducir al alumno al entendimiento de esta filosofía le da una base para su correcta aplicación al ejercer la profesión y poder gozar de los beneficios en los proyectos que participe. Según las encuestas, la efectividad de todas las actividades fue superior al $90 \%$, lo que demuestra el éxito de la metodología.

Palabras clave: Lean Construction; Enseñanza; Estudio de Caso; Sistema del Último Planificador

\section{ABSTRACT}

This paper aims to highlight the importance of teaching Lean Construction philosophy for civil engineering undergraduate students and give guidelines for its update on course design, taking into account that for effective learning the course must include real construction projects problems solving and knowing that students have very little or no professional experience. Therefore, it is essential to use tools that help the student to understand the problem and be able to develop analytical and critical thinking. In this sense, a very useful didactic tool is the use of case studies, since it makes the student understand the problems related to construction projects and how they can propose solutions through the application of Lean concepts. It is important to mention that the application of the Lean philosophy both in Peru and in the world is rapidly gaining ground; therefore, introducing the student to this philosophy gives a basis for its correct implementation. According to the surveys, the effectiveness of all activities was higher than $90 \%$, which demonstrates the success of the methodology.

Keywords: Lean Construction; Teaching; Case study; Last Planner System 


\section{La enseñanza de Lean Construction para alumnos en la formación de Ingenieros Civiles: \\ Una actualización del diseño del curso \\ Sofia Villagarcia Zegarra, Xavier Brioso}

\section{INTRODUCCIÓN}

La enseñanza de cursos de gestión en la carrera de ingeniería civil, que complementen el lado técnico de la formación de los alumnos, ha cobrado mucha importancia en los últimos años. Tradicionalmente estas habilidades se aprendían al momento de ejercer la profesión, cuando el profesional lidiaba con temas de manejo, organización, dirección de los recursos humanos, planificación de tareas y resolución de diversos problemas. Sin embargo, es importante reconocer que el ejercicio de la carrera de ingeniería de civil se desarrolla en un escenario complejo donde existe una elevada incertidumbre y variabilidad $[1,2]$ como consecuencia de las características propias de la construcción, como son:

- La gran cantidad de involucrados (cliente, supervisión, proveedores, subcontratista, etc.).

- Gran cantidad de especialidades que se dan en un mismo proyecto.

- La fase de construcción, muchas veces, parte de un diseño con omisiones y muchas veces incompleto, lo cual genera modificaciones a lo largo de la ejecución.

- La construcción es artesanal con elevado uso de mano de obra y bajo grado de industrialización.

Las características antes descritas hacen que sea indispensable que el profesional que lidere y que forma parte de los equipos tenga conocimientos de gestión que complemente el lado técnico para poder lograr el éxito en los proyectos tanto en el cumplimiento de costos como plazo [3].

Por otro lado, la filosofía Lean Construction tiene como fin principal maximizar el valor y reducir los desperdicios $[4,5]$, y reconoce que la construcción es un sistema complejo y variable $y$, por lo tanto, es importante su entendimiento para desarrollar herramientas que sirvan para optimizar y redefinir los procesos a fin de cumplir con los objetivos del proyecto. Por ello, en los últimos años ha crecido el interés de las empresas del rubro en conocer la filosofía y aplicarla en sus proyectos [6].

En este sentido, existe la necesidad de complementar la formación de los alumnos con cursos de gestión y, por otro lado, se tiene una alta demanda del mercado de profesionales con conocimientos de la filosofía lean, mediante la cual se reconoce la complejidad de los procesos constructivos y se proponen estrategias y principios para obtener proyectos con menos desperdicios y más valor. Por estos motivos, desde el año 2012 se imparte el curso "Gestión de la construcción sin pérdidas (Lean Construction) en la Pontificia Universidad Católica del Perú [7], asignatura que se viene actualizando acorde a las necesidades de la industria, la cual también están en constante actualización y muy interesada en aplicar la filosofía Lean [8].

Por todo lo anterior, este trabajo tiene como objetivo presentar pautas para la actualización del diseño del curso sobre "Lean Construction" de una manera tal que el alumno pueda aprender a desarrollar un pensamiento crítico y analítico acorde a lo que la industria demanda.

\section{MARCO TEÓRICO}

En los últimos años, de forma global en varias universidades del mundo se viene ofreciendo cursos sobre Lean Construction que proporcionan una comprensión de la filosofía Lean y las herramientas, técnicas y métodos que la constituyen $[9,10]$. 


\section{La enseñanza de Lean Construction para alumnos en la formación de Ingenieros Civiles: \\ Una actualización del diseño del curso \\ Sofia Villagarcia Zegarra, Xavier Brioso}

Si bien queda clara la importancia de enseñar esta disciplina, es también fundamental el diseño y actualización de la misma ya que es imprescindible que el alumno entienda primero la problemática de los proyectos para poder así entender la necesidad de la solución planteada desde la filosofía Lean. Por ello, es crucial tener en consideración que los alumnos tienen nula o muy poca experiencia profesional con lo cual es necesario el uso de herramientas didácticas adecuadas para el aprendizaje que contribuyan el entendimiento de los problemas y situaciones que se van a encontrar en proyectos reales.

En estas asignaturas se suelen implementar conferencias, talleres, simulaciones y períodos de discusión, y los temas cubiertos incluyen principios de Lean Construction, Lean Project Delivery System (LPDS), Last Planner System (LPS), planificación mediante trenes de actividades $\mathrm{y} / \mathrm{o}$ líneas de balance, métodos multicriterio de toma de decisiones, lecciones aprendidas y proyectos de fin de curso $[11,12]$. En el caso de la Pontificia Universidad Católica del Perú, entre el año 2012 y 2015, se implementaron, entre otras herramientas: (1) el uso del método multicriterio de toma de decisiones; (2) la simulación de reducción del tamaño del lote y flujo de una pieza; (3) el taller de sectorización y diseño del sistema de producción por flujo; (4) la simulación complejidad del diseño y construcción; (5) el taller Sesión de Pull Planning; (6) el taller de uso de la Carta de Balance para la mejora de flujo de conversión; (7) Elaboración de un Proyecto de Fin de curso [7].

Por otro lado, en diversas publicaciones sobre Lean Construction se demuestra que la enseñanza a través de estudios de caso es una manera de llevar al alumno de una forma entretenida a entender las situaciones y a buscar maneras de proponer soluciones para problemas reales planteados $[13,14]$.

\section{Building Information Modeling}

Se define Building Information Modeling (BIM) como la representación digital compartida de las características físicas y funcionales de cualquier objeto construido que forma una base confiable para las decisiones [15]. BIM es un término que se utiliza para describir herramientas, procesos y tecnologías que proporcionan documentación digital sobre un edificio, relacionados a su rendimiento, planificación, construcción y su funcionamiento [16]. BIM presenta los edificios virtualmente como modelos de construcción digitales que comprenden todo su ciclo de vida compuestos de objetos paramétricos con información [17]. Se ha demostrado que existe sinergia entre el Lean Construction y el BIM y que su uso simultáneo es una práctica que se viene extendiendo tanto en la industria como en la academia $[18,19]$.

\section{$\underline{\text { Tren de Actividades }}$}

El tren de actividades es una herramienta para administrar actividades repetitivas $y$ secuenciales, estructuras de edificaciones, tendido de tuberías, etc. [20]. En la comunidad Lean, al tren de actividades también se le denomina Takt Time Planning [21].

El objetivo del tren de trabajo es que se cumpla el flujo y cada paquete sea como un vagón que jale al siguiente. Luego se calculan las mediciones y se verifica que estén balanceadas por cada actividad, es decir, que aproximadamente tengan los mismos valores cada día. Con ello se afina la sectorización. Conociendo los rendimientos o productividades por cuadrilla se puede dimensionar el número de cuadrillas.

En un tren de actividades, éstas van conectadas como "vagones". Los pasos para seguir para desarrollar un Tren de Actividades: (1) Sectorizar el área de trabajo: Determinar áreas pequeñas y 


\section{La enseñanza de Lean Construction para alumnos en la formación de Ingenieros Civiles: \\ Una actualización del diseño del curso \\ Sofia Villagarcia Zegarra, Xavier Brioso}

la menor cantidad de repeticiones, generando la curva de aprendizaje; (2) Listar actividades necesarias; (3) Secuenciar las actividades (incluir colchones de tiempo de ser necesario); (4) Dimensionar recursos.

La sectorización está relacionada con la teoría de lotes de producción y lotes de transferencia, ya que al dividir el trabajo en sectores más pequeños estamos dividiendo nuestro lote de producción en lotes más pequeños que serán los que transferimos a las actividades siguientes (lotes de transferencia) [22].

Los trenes de trabajo aseguran el flujo y hacen que se obtenga beneficios de la curva de aprendizaje. Dan confiabilidad al planeamiento, ya que todos los días cada cuadrilla sabe lo que va a ejecutar y sus cargas de trabajo son similares.

\section{Líneas de balance}

Se define a la línea de balance como un método de planificación y programación gráfica que considera a la localización (sectorización) explícitamente como una dimensión, facilitando la planificación de recursos logrando mejores costos, menores riesgos en la programación y permanencia en el sector de trabajo de las cuadrillas [23, 24]. La localización (sector) se representa en el eje vertical, el eje horizontal representa al tiempo y las actividades o tareas se representan en las líneas de las gráficas. La línea de balance también se denomina línea de flujo si se diseña expresamente los flujos de sectores, mano de obra, equipos, materiales, información, trabajo previo y condiciones externas [23, 24]. La línea de balance es una herramienta alternativa al tren de actividades, ambas tienen ventajas y desventajas respecto de la otra y existe discusión sobre si alguna es mejor [25]. Con la misma información se puede generar los dos gráficos simultáneamente por lo que es muy recomendable utilizar software que automatice su generación. La línea de balance cada vez se utiliza más en la comunidad Lean, siendo una herramienta muy eficaz, tanto en la planificación como en la programación [21, 26].

\section{METODOLOGÍA}

\subsection{Objetivos}

Para la actualización del diseño del curso se plantearon cinco objetivos principales:

- Entender un proyecto de construcción desde un enfoque sistémico cuyo éxito depende de las diversas partes involucradas: cliente, proyectistas, constructores, proveedores, normatividad y formalización del sector.

- Identificar las fuentes de pérdidas en las diversas etapas de un proyecto de construcción: Ingeniería, procura (abastecimiento) y construcción, operación.

- Usar apropiadamente los conceptos, técnicas y herramientas Lean Construction aplicados en los proyectos de construcción e identificar las variables con mayor incidencia para el éxito de un proyecto de construcción.

- Desarrollar criterios para el control y mejoramiento de la productividad en obras.

- Implementar un sistema de indicadores enfocados al mejoramiento de los procesos constructivos, así como el cálculo de mediciones cuantitativas y cualitativas para el mejoramiento de los procesos constructivos.

\subsection{Metodología}

Para lograr cumplir estos objetivos se identificó una metodología apropiada para la actualización de la enseñanza, teniendo en cuenta que para aprender el alumno necesitaba entender lo que se vive en el día a día de una obra en construcción, teniendo en cuenta que los alumnos no tenían experiencia profesional. 


\section{La enseñanza de Lean Construction para alumnos en la formación de Ingenieros Civiles: \\ Una actualización del diseño del curso \\ Sofia Villagarcia Zegarra, Xavier Brioso}

Para ello se utilizaron varias formas de compartir el conocimiento:

- Clases Teórico / prácticas: es decir después de la teoría siempre debe existir un trabajo práctico para poder consolidar el conocimiento

- Discusiones en clase de problemas reales de obras

- Lecturas: que se dejaban para la casa y se discutían en clase.

- Trabajos en equipo que se desarrollaban durante la clase después de impartida la parte teórica puedan trabajar en grupos e iniciar después una discusión de clase.

- Proyectos prácticos específicos con visitas a obras de construcción para trabajar en equipo y presentar los resultados en clase. La idea era que el alumno adquiera conocimientos y competencias a través de la elaboración de proyectos que dan respuesta a problemas reales.

- Proyectos dirigidos con acompañamiento de asistentes de docencia.

- Proyectos en que se implemente la tecnología BIM y la filosofía Lean Construction.

- Videos y películas relacionadas con lean, etc.

\subsection{Encuestas}

Para validar la metodología propuesta se utilizó las encuestas anónimas tomadas a los alumnos sobre el desarrollo del curso. En este artículo se presentan los resultados de las últimas cinco encuestas aplicadas en la escuela.

\section{RESULTADOS}

\subsection{Partes principales}

\subsubsection{Desperdicio y Pérdidas}

El curso parte del entendimiento de lo que es desperdicio y pérdidas y de las diferentes formas que esta se presenta en el proceso constructivo. Este concepto es primordial ya que Lean Construction se focaliza en su eliminación. Para el mejor entendimiento de este concepto se utilizan fotos de desperdicios físicos y de otros tipos de desperdicios y se les hace discutir en grupos acerca de las fotos y que problemas identifican. Esta dinámica es interesante por que promueve la discusión entre los miembros del equipo y incentiva el análisis crítico, ya que no solo identifican los problemas sino también discuten acerca de lo que pasó y por qué sucedió.

También es importante identificar en qué momento se origina el desperdicio, el cual muchas veces se puede iniciar en etapas previas, como por ejemplo en un proyecto mal definido con omisiones; en este caso, el presentar ejemplos de incompatibilidades $u$ omisiones de proyectos reales ayuda al alumno a entender el impacto de un proyecto mal desarrollado en el costo y plazo de la obra. También podría suceder que el desperdicio se haya originado en el proveedor, y la idea es que según sea el caso se debe brindar ejemplos reales e iniciar una discusión acerca del tema. Como se indicó anteriormente, el análisis de casos reales es una buena práctica ya que abre la curiosidad de los alumnos y los motiva a prestar más atención que cuando la clase es teórica.

\subsubsection{Lean Construction: Conceptos y Origen}

Este es un capitulo muy importante donde se tiene que dejar claro los conceptos y los objetivos de la filosofía Lean. Las herramientas de enseñanza para esta parte son variadas. Son importantes el desarrollo de la teoría en clase lo cual se deben complementar con lecturas que contengan conceptos claros sobre Lean. 


\section{La enseñanza de Lean Construction para alumnos en la formación de Ingenieros Civiles: \\ Una actualización del diseño del curso \\ Sofia Villagarcia Zegarra, Xavier Brioso}

El entendimiento del origen de la filosofía es fundamental y lleva al alumno a entender el contexto y la industria en que inicialmente se desarrolló la filosofía, el porqué del éxito de la misma $y$, el tiempo que demoró en implementarse y desarrollarse.

De esta manera el alumno entiende que Lean Construction no es solo un conjunto de herramientas, ni una receta, es una filosofía con un enfoque holístico y por lo tanto involucra cambios de mentalidad y forma de trabajo. Conseguir cambios en los involucrados es el resultado de liderazgo, constancia y tiempo. Se debe tener claro que la única forma de implementarla correctamente es entendiendo los conceptos y premisas en las que se basa, ya que a partir de este entendimiento el profesional que lo implemente va a poder desarrollar sus propias soluciones o herramientas y adaptarlas a la industria de la construcción, es importante recordar que Lean nace en la industria automotriz y que se adapta a la construcción [3, 4].

Se pretende que con las lecturas se abran espacios para discusiones y trabajos en clase para asegurarse que los conceptos principales se fijen. Como se mencionó anteriormente, el uso de dinámicas y juegos de simulación para entender el impacto de la variabilidad en los procesos son bastante útiles. En la literatura sobre Lean podemos encontrar varias herramientas, talleres y juegos de simulación que exploran estos conceptos $[9,10,11,12]$.

\subsubsection{Planeamiento}

En cuanto a la planificación, Lean se basa en un enfoque sistémico de la producción e incluye todas las partes que participan en los procesos y las relaciones que se dan entre ellas, se entiende que para tener éxito en los proyectos se debe partir de un planeamiento realizado por las personas que intervienen en el trabajo. El sistema de planeamiento Lean se basa en "jalar" la producción y no de "empujarla" como tradicionalmente sucede, propone un planeamiento Ilamado Last Planner System o Sistema del Ultimo planificador y se plantean tres niveles de planificación [27, 28, 29]:

- Plan maestro: se planifica las fases por hitos y se parte de la premisa que siendo la construcción un entorno tan variable no es muy útil hacer un planeamiento muy detallado de largo plazo porque lo más probable es que en pocas semanas se comience a desfasar por la naturaleza misma de la construcción y por la cantidad de procesos y personas involucradas. El principal objetivo de este planeamiento es el cumplimiento del plazo total e identificar hitos claves para garantizar el cumplimiento de este plazo.

- Plan a mediano plazo: que parte del anterior, pero contiene más detalle y se suele trabajar en una ventana de 3 a 5 semanas dependiendo de la velocidad de la logística. Este nivel de planeamiento llamado también "Lookahead" tiene como objetivo asegurar el plan maestro y descomponer sus actividades en paquetes de trabajo y operaciones, y desarrollar métodos detallados para ejecutar el trabajo identificando las principales restricciones (prerrequisitos) y responsables del levantamiento de estas.

- Plan semanal: es el más detallado de los tres. Se determina a detalle las cantidades que se necesitan avanzar y se calculan las cuadrillas que harán el trabajo diario.

\section{Planeamiento Pull}

La enseñanza de la forma de realizar planificación se debe impartir usando la metodología de trabajo en equipo ya que así es 


\section{La enseñanza de Lean Construction para alumnos en la formación de Ingenieros Civiles: \\ Una actualización del diseño del curso \\ Sofia Villagarcia Zegarra, Xavier Brioso}

como sucede en los proyectos reales haciendo intervenir a los involucrados y que participen colaborativamente.

Como ejemplo de una dinámica grupal se les otorga a los alumnos un proyecto que consiste en un estudio de caso donde se les explica lo que se quiere construir, la problemática, se les entrega planos o croquis del problema y también se les puede brindar una tabla donde se listan las actividades principales $y$ sus duraciones y dependencias entre ellas $[12,30]$. Cada miembro del equipo representa a un responsable (ver Tabla 1). La idea es entregar la información necesaria para que cada alumno entienda el problema y la secuencia de actividades que se necesitan realizar.
IT. RESPONSABLE UBICACIÓN

\begin{tabular}{|c|c|c|c|c|c|}
\hline 1 & Contratista & & $\begin{array}{l}\text { Planos de Arquitectura e } \\
\text { Instalaciones }\end{array}$ & $\begin{array}{l}\text { información } \\
\text { Proveedores }\end{array}$ & 0 \\
\hline 2 & Contratista & AZOTEA & $\begin{array}{c}\text { Pases en losa maciza para ductos } \\
\text { de ventilación (blowers) de } 6 \\
\text { máquinas. }\end{array}$ & 1 & 7 \\
\hline 4 & Contratista & AZOTEA & $\begin{array}{c}\text { Bases para equipos de Blowers en } \\
\text { azoteas }\end{array}$ & 1,2 & 3 \\
\hline 5 & Contratista & NIVEL 9 & $\begin{array}{l}\text { Falso cielo raso (FCR) con drywall } \\
\text { después de instalados los ductos }\end{array}$ & 1,12 & 5 \\
\hline 6 & Contratista & NIVEL 9 & Pintado paredes de FCR NIVEL 9 & $1,5,9$ & 10 \\
\hline 8 & $\begin{array}{l}\text { SC de Piso } \\
\text { Epóxico }\end{array}$ & NIVEL 9 & $\begin{array}{l}\text { Ejecución de piso epóxico en salas } \\
\text { de donde están las campanas }\end{array}$ & 1,6 & 3 \\
\hline 9 & $\begin{array}{l}\text { Proveedor de } \\
\quad \text { IIEE }\end{array}$ & NIVEL 9 & $\begin{array}{l}\text { Puntos de Instalaciones eléctricas } \\
\text { para campanas - NIVEL } 9\end{array}$ & 1 & 1 \\
\hline 10 & $\begin{array}{l}\text { Proveedor de } \\
\quad \text { IIEE }\end{array}$ & AZOTEA & $\begin{array}{l}\text { Puntos pata instalaciones } \\
\text { eléctricas para Blowers - Azotea }\end{array}$ & 1 & 1 \\
\hline 12 & $\begin{array}{l}\text { Proveedor } \\
\text { Campanas }\end{array}$ & NIVEL 9 & Instalación de ductos en FCR & 1,2 & 10 \\
\hline 13 & $\begin{array}{l}\text { Proveedor } \\
\text { Campanas }\end{array}$ & NIVEL 9 & Instalación de ductos Azotea & 1,2 & 10 \\
\hline 14 & $\begin{array}{l}\text { Proveedor } \\
\text { Campanas }\end{array}$ & NIVEL 9 & Instalación de campanas & $1,6,8$ & 14 \\
\hline 15 & $\begin{array}{l}\text { Proveedor } \\
\text { Campanas }\end{array}$ & AZOTEA & Instalación de Blowers - Azotea & $1,4,10,13$ & 7 \\
\hline
\end{tabular}

Tabla 1: Ejemplo de actividades principales, dependencias y duraciones 
Se puede trabajar en una pizarra o papelógrafo y cada actividad debe ser representada con un post-it de color diferente según responsable. Cada responsable identifica sus actividades y las coloca en un post-it con los siguientes datos:

Actividad

\begin{tabular}{l|l}
\hline Actividad Previa & Duración \\
\hline
\end{tabular}

Fig. 1: Datos de Post-it

Cuando ya están realizados los post-it cada responsable coloca en la pizarra o papelógrafo los post-it en una secuencia lógica en reversa. Una vez que todas las tareas estén en un orden lógico el equipo revisa la secuencia. Hasta este punto no hay fechas en las secuencias. Solo se han considerado las tareas y se han consignado duraciones para cada una. La idea es revisar el flujo y tratar de establecer la mejor secuencia para el problema dado y tratar de reducir el tiempo de duración de todo el proceso. Cada alternativa se representa mediante un tren de actividades genérico.

Este es un trabajo que hace que el alumno perciba que para lograr el mejor resultado deben integrarse como equipo, la idea no es que una actividad termine más rápido, sino que toda la secuencia sea la más optima desarrollando capacidad de análisis, capacidad de negociación, por que van a tener que argumentar para plantear si existe alguna mejor manera de resolver el problema en beneficio del proceso. Además, les da una percepción de lo que sucede en los proyectos reales ya que estos no solo dependen de un solo actor sino de varios y en la integración de los mismos está el éxito del proyecto. Si bien esta actividad se puede usar para hacer el planeamiento de un proceso, también se puede usar para hacer el plan maestro, para lo cual se deben definir las fases de trabajo y fechas de entrega de cada hito.

De manera similar, este trabajo con post-its se debe desarrollar en las obras para realizar la planificación maestra y requiere de la participación de los principales actores.

Para los otros niveles de planeamiento tales como el Lookahead, el plan semanal y los planes diarios, se pueden usar las herramientas descritas con un mayor nivel de detalle aprovechando la experiencia previa, lo cual facilita la identificación de los incumplimientos de la programación y la adopción de las medidas correctivas más precisas.

\section{Tren de Actividades, Líneas de Balance y BIM}

En el caso de trenes de actividades, la enseñanza se debe hacer a través de ejercicios prácticos. Por ejemplo, se les puede entregar una planta típica de un edificio, de preferencia, los proyectos deben estar modelados con BIM pues se facilita el mapeo de procesos por la sinergia existente entre Lean y BIM [31, 32]. Luego los alumnos tienen que proponer la mejor sectorización buscando tener áreas o porciones de trabajo similares que se pueden ejecutar en un día para poder implementar el tren de actividades, teniendo en cuenta las consideraciones estructurales y la cantidad de días de la semana para completar un piso. Esto se puede hacer a través de prácticas dirigidas 0 después de haber resuelto en la hora de clase un ejemplo con varias alternativas y evaluando la mejor solución. En la figura 1 se muestra un tren de actividades genérico que se puede variar durante las discusiones, y en la figura 2 se observa una sectorización propuesta sobre un modelo BIM. 
La enseñanza de Lean Construction para alumnos en la formación de Ingenieros Civiles:

Una actualización del diseño del curso

Sofia Villagarcia Zegarra, Xavier Brioso

\begin{tabular}{|c|c|c|c|c|c|c|c|c|c|c|c|c|c|c|}
\hline 芯 & Lun & Mar & Mie & Jue & Vie & Sab & Dom & Lun & Mar & Mie & Jue & Vie & Sab & Dom \\
\hline $\mathbb{\digamma}$ & D1 & D2 & D3 & D4 & D5 & D6 & D7 & D8 & D9 & D10 & D11 & D12 & D13 & D14 \\
\hline T1 & S1 & S2 & S3 & S4 & S5 & S6 & & S7 & S8 & S9 & $\mathrm{S} 10$ & S11 & S12 & \\
\hline T2 & & S1 & S2 & S3 & S4 & S5 & & S6 & S7 & S8 & S9 & S10 & S11 & \\
\hline T3 & & & $\mathrm{S} 1$ & $\mathrm{~S} 2$ & S3 & $\mathrm{S} 4$ & & S5 & S6 & $\mathrm{S} 7$ & S8 & S9 & $\mathrm{S} 10$ & \\
\hline T4 & & & & S1 & S2 & S3 & & S4 & S5 & S6 & S7 & S8 & S9 & \\
\hline T5 & & & & & S1 & $\mathrm{S} 2$ & & S3 & S4 & S5 & S6 & S7 & S8 & \\
\hline T6 & & & & & & S1 & & $\mathrm{S} 2$ & S3 & $\mathrm{S} 4$ & S5 & S6 & S7 & \\
\hline T7 & & & & & & & & S1 & S2 & S3 & S4 & S5 & S6 & \\
\hline T8 & & & & & & & & & S1 & $\mathrm{S} 2$ & S3 & S4 & S5 & \\
\hline T9 & & & & & & & & & & S1 & S2 & S3 & S4 & \\
\hline T10 & & & & & & & & & & & S1 & S2 & S3 & \\
\hline T11 & & & & & & & & & & & & S1 & S2 & \\
\hline
\end{tabular}

Fig. 2: Alternativa de tren de Actividades

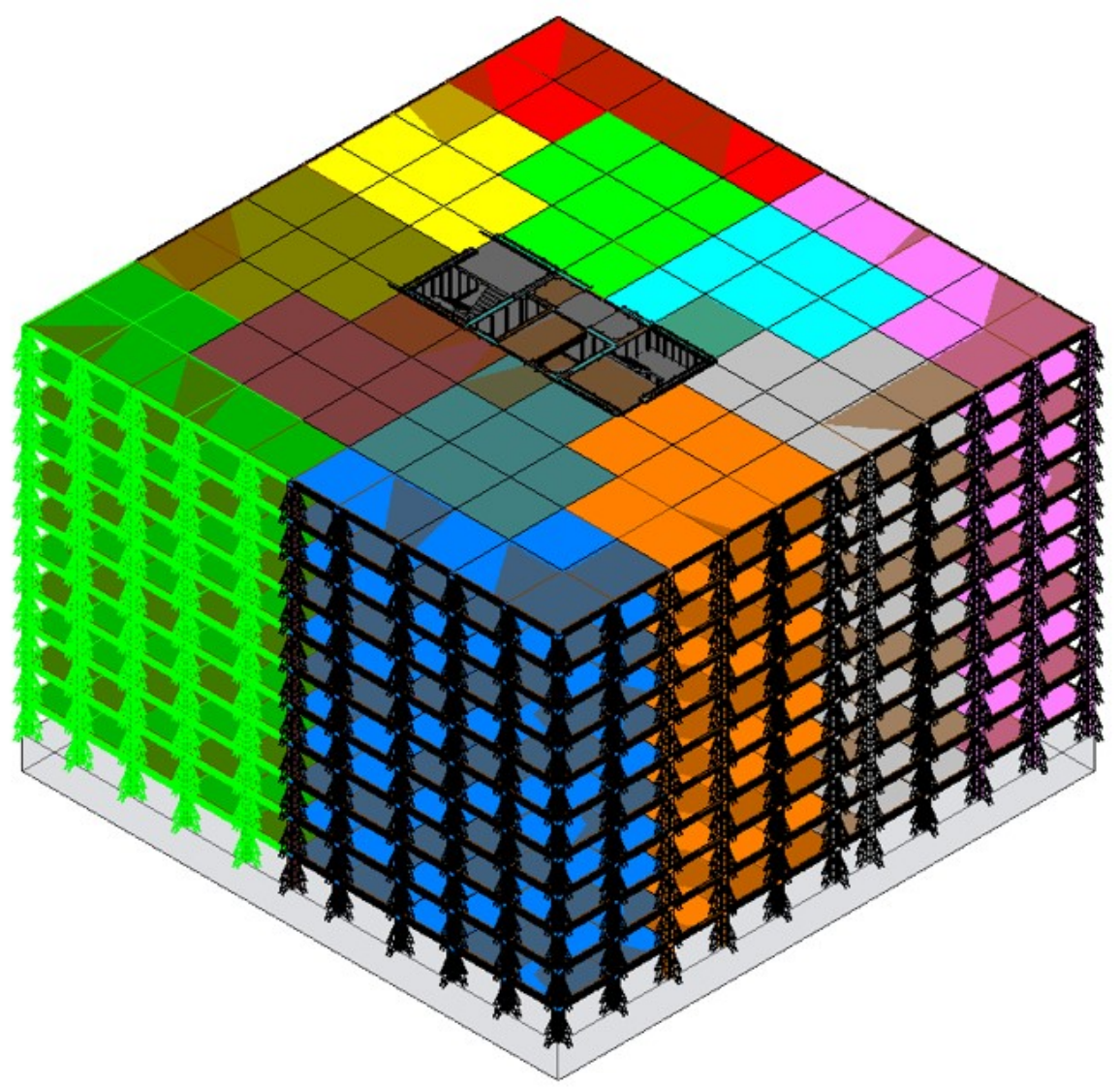

Fig. 3: Sectorización mediante modelos BIM

Advances in Building Education / Innovación Educativa en Edificación | ISSN: 2530-7940 | http://polired.upm.es/index.php/abe

| Cod. 0088 | Septiembre-Diciembre 2020 | Vol. 4. № 3 | pp. 09/22 | 
Como se indicó anteriormente, generar las líneas de balance correspondientes a cada tren de actividades es un buen ejercicio para que los alumnos ejerciten dos formas muy potentes de visualizar y entender cómo se van a desarrollar los trabajos en cada sector a lo largo del tiempo.
En la comunidad Lean se ha determinado que cada método tiene sus ventajas y desventajas, pero que en general, es mejor generar ambas. En la figura 4, se muestra la línea balance correspondiente al tren de actividades mostrado en la figura 2.

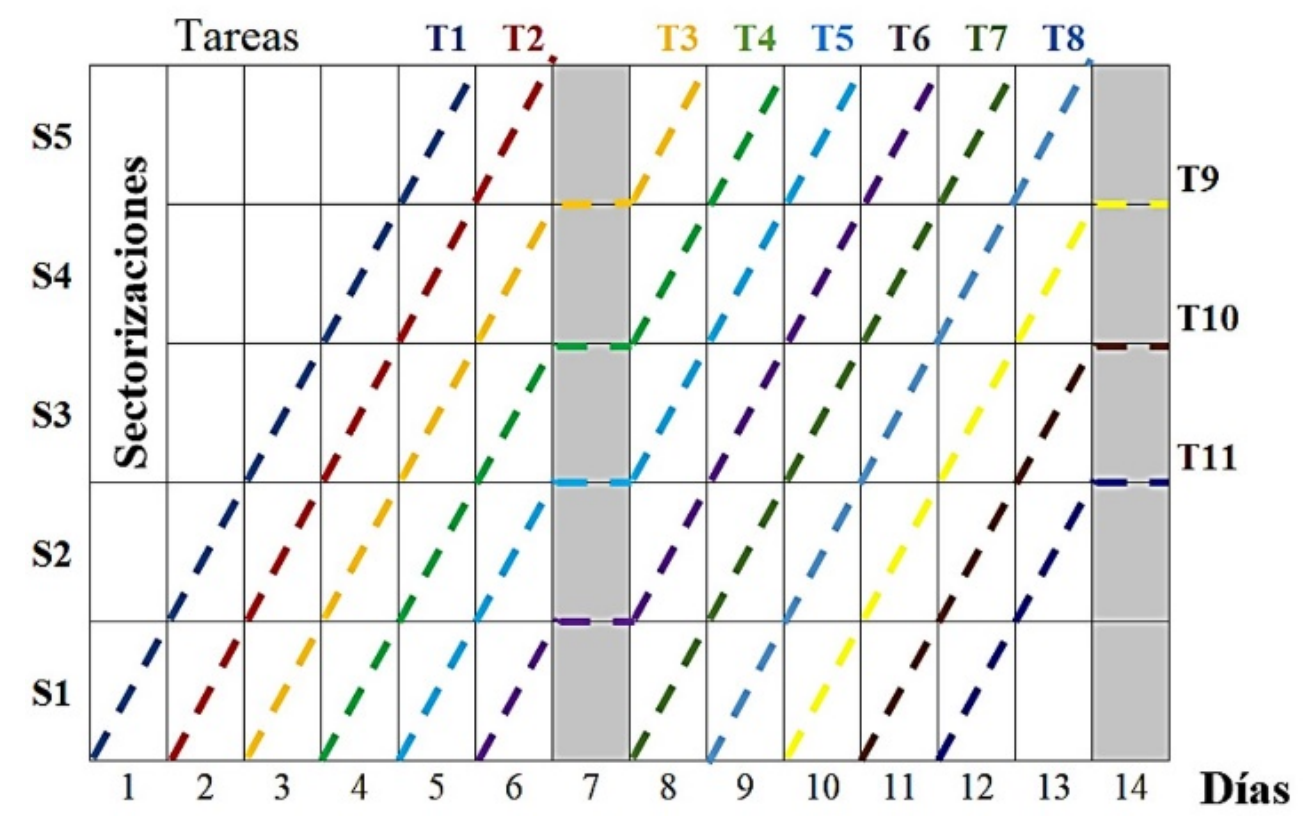

Fig. 4: Línea de balance de una alternativa de Tren de Actividades

\subsubsection{Encuesta}

La tabla 2 muestra los resultados promedio de la encuesta anónima de opinión tomada a los alumnos en las últimas cinco veces en que se ofreció la asignatura. Se puede apreciar que la efectividad general promedio obtenida fue de $90 \%$ y que el promedio obtenido por todos los profesores de la escuela fue de $84 \%$. La misma tendencia se aprecia en las diferentes secciones de la encuesta, tales como: (A) Dominio disciplinar y apertura al diálogo (96\% vs. $91 \%$ ); (B) Planificación del aprendizaje (93\% vs. $88 \%$ ); (C) Metodología y uso de recursos ( $91 \%$ vs. $86 \%$ ); (D) Evaluación del aprendizaje (90\% vs. $86 \%$ ); y (E) Comunicación e interacción (92\% vs. $88 \%)$.
Por otro lado, la efectividad general promedio obtenida en el período anterior (2012-2015) fue de $83 \%$ [7], por lo que el $90 \%$ obtenido ahora nos muestra que existe una mejor valoración del estudiantado por los nuevos enfoques. Asimismo, se obtuvieron comentarios favorables de los encuestados, y también propuestas de mejora, tales como la contratación de proveedores de software especializado que centralicen las herramientas Lean partiendo del modelo BIM, mayores sesiones con los profesionales de la industria, y visitas presenciales 0 virtuales a empresas que implementan la filosofía Lean Construction. Todos estos resultados validan lo exitosa que resultó la metodología propuesta. 


\begin{tabular}{lcc}
\hline Sección encuesta & $\begin{array}{c}\text { Efectividad promedio } \\
\text { de la Escuela (\%) }\end{array}$ & $\begin{array}{c}\text { Efectividad promedio } \\
\text { del profesor (\%) }\end{array}$ \\
\hline A. Dominio disciplinar y apertura al diálogo. & 91 & 96 \\
\hline B. Planificación del aprendizaje & 88 & 93 \\
\hline C. Metodología y uso de recursos & 86 & 91 \\
\hline D. Evaluación del aprendizaje & 86 & 90 \\
\hline E. Comunicación e interacción & 88 & 92 \\
\hline F. Calificación general & 84 & 90 \\
\hline
\end{tabular}

Tabla 2. Resultados promedio de últimas cinco encuestas

\section{CONCLUSIONES}

A lo largo del presente trabajo, se ha demostrado que el Lean Construction es una filosofía importante a impartir en la formación de ingenieros civiles, y que es valorada por la industria, los docentes y por los mismos alumnos. Sin embargo, para entender sus conceptos fundamentales es recomendable que se incluya la problemática de las obras con estudios de caso reales, de preferencia usando la tecnología BIM, lo cual incrementa el entendimiento y visualización del proyecto y de las herramientas Lean, entre ellas, las que conforman el Sistema del Último Planificador, el tren de actividades y la línea de balance. Las discusiones en clase y los trabajos en equipo de forma colaborativa, además de los talleres y simulaciones, son esenciales para que los alumnos puedan profundizar en el tema, y desarrollar y ampliar su capacidad de análisis y solución de problemas. Los resultados obtenidos en las encuestas con una efectividad superior al $90 \%$ demuestra el éxito de la actualización del diseño del curso, por lo que se debe seguir buscando oportunidades de nuevas innovaciones según las últimas tendencias de la industria de la arquitectura, ingeniería y construcción, integrando de manera flexible la filosofía Lean Construction, el BIM y el Project Management [33].

\section{REFERENCIAS}

[1] Oprach, S., Steuer, D., Krichbaum, V. \& Haghsheno, S. (2019). Smart Data - Dealing With Task Complexity in Construction Scheduling. 27th Annual Conference of the International Group for Lean Construction (IGLC). Dublin, Ireland, 3-5 Jul 2019. pp 347-358.

[2] Kortenko, S., Koskela, L., Tzortzopoulos, P. \& Haghsheno, S. (2020). Negative Effects of Design-Bid-Build Procurement on Construction Projects. 28th Annual Conference of the International Group for Lean Construction (IGLC). Berkeley, California, USA, 6-10 Jul 2020. pp 733-744.

[3] Brioso, X. (2015), El Análisis de la Construcción sin Pérdidas (Lean Construction) y su relación con el Project \& Construction Management: Propuesta de Regulación en 


\section{La enseñanza de Lean Construction para alumnos en la formación de Ingenieros Civiles: \\ Una actualización del diseño del curso \\ Sofia Villagarcia Zegarra, Xavier Brioso}

España y su Inclusión en la Ley de la Ordenación de la Edificación. PhD thesis. Technical University of Madrid, Spain, 2015.

[4] Koskela, L. (2000). An Exploration towards a Production Theory and its Application to Construction. PhD Dissertation, VTT Building Technology, Espoo, Finland.

[5] Brioso, X. \& Humero, A. (2017). Incorporating Lean Construction agent into the Building Standards Act: the Spanish case study. Organization, Technology and Management in Construction: an International Journal, 8(1), pp. 1511-1517.

[6] Demirkesen, S. , Wachter, N. , Oprach, S. \& Haghsheno, S. (2019). Identifying Barriers in Lean Implementation in the Construction Industry. 27th Annual Conference of the International Group for Lean Construction (IGLC). Dublin, Ireland, 3-5 Jul 2019. pp 157-168

[7] Brioso, X. (2015). Teaching Lean Construction: Pontifical Catholic University of Peru Training Course in Lean Project \& Construction Management. Procedia Engineering, 123 (2015) 85 - 93.

[8] Ghannoum, C., Antar, S., Daoud, Y. and Hamzeh, F. (2019). Are Construction Clients Ready to Lead the Lean Initiative? Lean Construction Journal, 2019(1), pp. 105-120.

[9] Tsao, C. C. Y., Alves, T. d. C. L. \& Mitropoulos, P. T. (2012). Different Perspectives on Teaching Lean Construciton. 20th Annual Conference of the International Group for Lean Construction. San Diego, USA.

[10] Alves, T.D.C.L., Milberg, C. \& Walsh, K.D. (2010). Exploring Lean Construction Practice, Research, and Education. 18th Annual Conference of the International Group for Lean Construction. Haifa, Israel.
[11] Nofera, W., Abdelhamid, T. \& Lahouti, A. (2015). Teaching Lean Construction for University Student(s), Lean Construction Journal, 2015 Issue. pp 34-44.

[12] Tsao, C.C., Draper, J. \& Howell, G.A. (2014). An Overview, Analysis, and Facilitation Tips for Simulations That Support and Simulate Pull Planning. 22nd Annual Conference of the International Group for Lean Construction. Oslo, Norway.

[13] Devkar, G., Trivedi, J. \& Pandit, D. (2019). Teaching Target Value Design: A Simulation. 27th Annual Conference of the International Group for Lean Construction (IGLC). Dublin, Ireland, 3-5 Jul 2019. pp 479-490.

[14] Brioso, X., Humero, A. and CalderonHernandez, C. (2018). Teaching how to integrate Last Planner System and the Safety and Health Management System. ABE (Advances in Building Education / Innovación Educativa en la Edificación), 2 (1), pp. 12-30.

[15] International Standards Office (2018). ISO 19650-1:2018. Organization and digitization of information about buildings and civil engineering works, including building information modelling (BIM) - Information management using building information modelling - Part 1: Concepts and principles. Geneva: ISO.

[16] Sacks, R., Eastman, C., Lee, G., Teicholz, P. (2018). BIM Handbook: A Guide to Building Information Modeling for Owners, Designers, Engineers, Contractors, and Facility Managers. (2018) Wiley.

[17] Volk, R., Stengel, J. and Schultmann, F. (2014). Building Information Modeling for Existing Buildings - Literature Review and Future Needs. Automation in Construction, 38,109-127.

[18] Sacks, R., Koskela, L., Dave, B., and Owen, R. (2010). Interaction of Lean and Building Information Modeling in Construction. Journal of 


\section{La enseñanza de Lean Construction para alumnos en la formación de Ingenieros Civiles: \\ Una actualización del diseño del curso \\ Sofia Villagarcia Zegarra, Xavier Brioso}

Construction Engineering and Management, 2010, 136(9): 968-980.

[19] Murguia, D., Brioso, X., Ruiz-Conejo, L. and Fernandez, L. (2017). Process Integration Framework for the Design Phase of a Residential Building. Procedia Engineering, 196, pp. 462469.

[20] Brioso, X., Murguía, D. \& Urbina, A. (2017). Comparing three scheduling methods using BIM models in the Last Planner System. Organization, Technology and Management in Construction: an International Journal, 9 (2017), Issue 1, pp. 1604-1614.

[21] Brioso, X., Humero, A. \& Calampa, S. (2016). Comparing Point-to-Point Precedence Relations and Location-Based Management System in Last Planner System: A Housing Project of Highly Repetitive Processes Case Study. Procedia Engineering, 164 (2016), pp. 12-19.

[22] Guzman, A (2014). Aplicación de la filosofía Lean Construction en la Planificación, Programación, Ejecución y Control de Proyectos. Tesis de Ingeniería Civil. Pontificia Universidad Católica del Perú.

[23] Seppänen, O., Ballard, G., \& Pesonen, S. (2010). The combination of last planner system and location-based management system. Lean Construction Journal, 2010 Issue, pp. 43-54.

[24] Seppänen, O., Evinger, J., \& Mouflard, C. (2013). Comparison of LBMS schedule forecasts to actual progress. 21st Annual Conference of the International Group for Lean Construction 2013 Fortaleza, Brazil, pp. 506-515.

[25] Brioso, X., Murguia, D. y Urbina, A. (2017). Teaching Takt-Time, Flowline, and Point-to-Point Precedence Relations: A Peruvian Case Study. Procedia Engineering, 196, pp. 666-673.
[26] Frandson, A. G., Seppänen, O. \& Tommelein, I. D. (2015). Comparison Between Location Based Management and Takt Time Planning. 23rd Annual Conference of the International Group for Lean Construction. Perth, Australia, 29-31 Jul 2015. pp 3-12.

[27] Ballard, G. (2000). The Last Planner System of Production Control, Ph.D. Disser-tation, School of Civil Engineering., Univertity of Birmingham, U.K., May, 192 pp.

[28] Ballard, G. and Howell, G. (2003). An update on Last Planner. 11th Annu. Conf. Int. Gr. Lean Constr., pp. 1-10, 2003.

[29] Ballard, G. and Tommelein, I. (2016). Current Process Benchmarket for the Last Planner System. Lean Construction Journal, 2016(1), pp. 57-89.

[30] Tvedt, I. M. (2020). Divergent Beliefs About Productivity Despite Concurrent Engineering and Pull Planning, a Case Study. 28th Annual Conference of the International Group for Lean Construction (IGLC). Berkeley, California, USA, 6-10 Jul 2020. pp 301-312.

[31] Ramalingam, S. (2018), Mapping of BIM Process for Teaching Lean. 26th Annual Conference of the International Group for Lean Construction. Chennai, India, 18-20 Jul 2018. pp 1291-1301.

[32] Murguia, D. and Brioso, X. (2017). Using Choosing by Advantages and 4D Models to Select the Best Construction-Flow Option in a Residential Building. Procedia Engineering, 196, pp. 470-477.

[33] Brioso, X. (2015). Integrating ISO 21500 Guidance on Project Management, Lean Construction, and PMBOK. Procedia Engineering, 123 (2015), pp. 76 - 84. 